\title{
Study of fluorescense for cryogenic fine-grain emulsion
}

\author{
H. Ichiki*, T. Naka, T. Shiraishi \\ Nagoya University \\ Furo-cho, Chikusa-ku, Nagoya 464-8601 JAPAN \\ E-mail: ichikidflab.phys.nagova-u.ac.jp
}

\begin{abstract}
The nuclear emulsion is known as a solid detector which consists of gelatine and $\mathrm{AgBr}$ crystals with several mol\% iodine (hereinafter, $\mathrm{AgBr} \cdot \mathrm{I}$ ), and records charged particle's track as a ligned silver grains. Electrons generated through the ionization by the penetrating charged particles react with interstitial silver ions in the crystal, and they become latent images. On the other hand, it is thought that iodine contained in the $\mathrm{AgBr}$.I crystal works as hole trap, and photons are emitted when the holes and electrons recombine. There are several reports that photon emission rate of silver halide crystals increased at cryogenic temperature. In those studies, they usually use light source or rubbing the sample to excite electrons. The enhancement is also expected with the excitation by charged particles, and we started basic research to use the nuclear emulsion as a scintillation detector. In this paper, we will report about the result of detecting photons from fine grain emulsion caused by $\beta$-ray at cryogenic temperature (77K) for the first time.
\end{abstract}

The 3rd International Symposium on "Quest for the Origin of Particles and the Universe" 5-7 January 2017

Nagoya University, Japan

\footnotetext{
* Speaker.
} 


\section{Introduction}

The nuclear emulsion is a solid detector which consists of gelatine and $\mathrm{AgBr}$ crystals containing several mol\% iodine (hereinafter, $\mathrm{AgBr} \cdot \mathrm{I}$ ) . The nuclear emulsion has been attracting attentions, because it can record the particle track with submicron spatial resolution as a row of silver grains through the development process. When a charged particle penetrates the crystals, electrons are generated by ionization and trapped on the crystal surface. Three relaxation processes of electrons are supposed: Ag core formation (reaction of electrons and interstitial silver ions), photon emission (recombination electrons and holes, Fig.WI), and the lattice vibration by phonons. At room temperature, $\mathrm{Ag}$ core formation process and the lattice vibration process are dominant, so it is hard to detect photon emission. However at cryogenic temperature, those processes are suppressed and photon emission process become dominant. Several researchers, e.g. S. Takada, report about this tendency for silver halide powder[四. Generally, they use a light source or rubbing to excite electrons to measure such photon emission process enhancement, though we use charged particles. If photon emission caused by a charged particle is sufficient intensity, nuclear emulsion can be a scintillation detector. In order to investigate this possibility, we try to detect photons from nuclear emulsion caused by $\beta$ rays at liquid nitrogen temperature with photodetector.

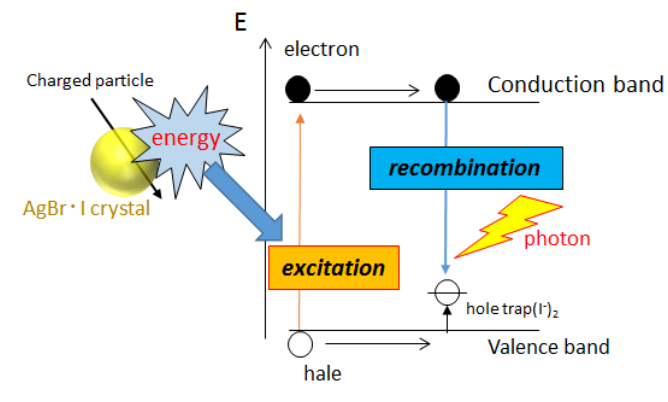

Figure 1: Schematic of photon emission process Electrons and holes are generated by ionization by the penetrating charged particles. Iodine capture hole and meet with electron, photon emission occur by recombination electron and hole.

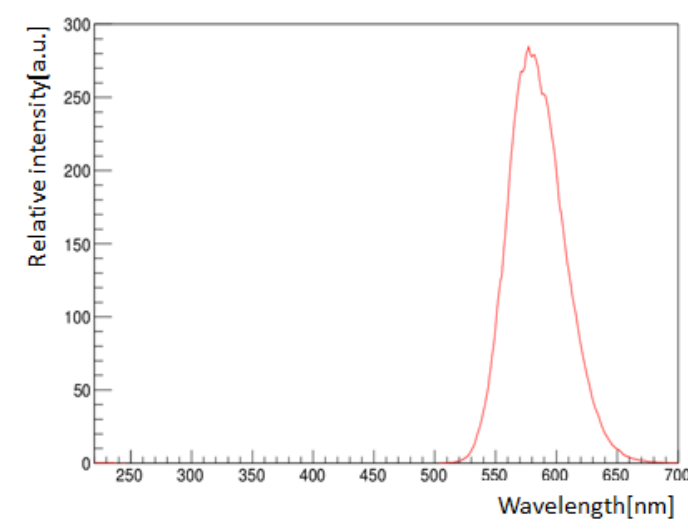

Figure 2: Emission spectrum of the emulsion with $40 \mathrm{~nm}$ crystals

The wavelength peak of the emission light was measured as $570 \mathrm{~nm}$ using a spectrometer.

\section{The pre-checks on photon emission enhancement at cryogenic temperature}

Here, we describe several test results done before starting this experiment. The nuclear emulsions were soaked in liquid nitrogen then exposed samples to fluorescent lamp and human eye observation whether light emission occur or not. Emulsions having crystal sizes of $20 \mathrm{~nm}, 40 \mathrm{~nm}$ and the OPERA film [[] were used. As a result, light emission (phosphorescence) was confirmed in all the samples, and we decided to use the nuclear emulsions with $40 \mathrm{~nm}$ crystals called NIT (Nano Imaging Tracker) . The wavelength peak of the emission light was measured as $570 \mathrm{~nm}$ using a spectrometer (Fig.D). 


\section{The photon emission measurement}

The measurement setup and information about used samples and radiation source are shown in Fig.[3 and TableW. We used ${ }^{90} \mathrm{Sr}$ as radiation source (hereinafter, RI) and NIT as a main sample (hereinafter, Film), and gelatine film as a reference(hereinafter, Gel.) since approximately $60 \%$ of Film consist of gelatin.

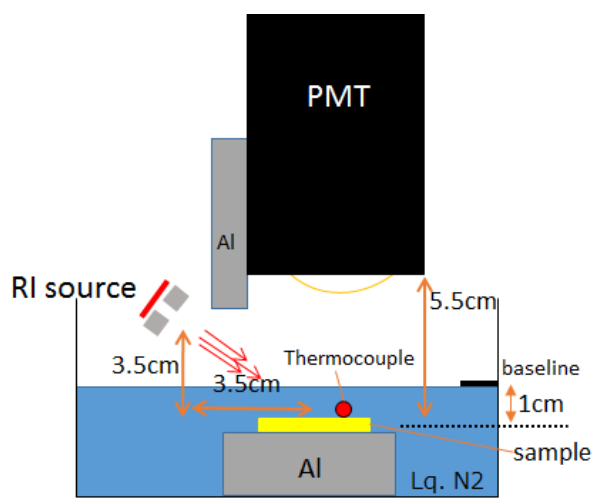

Figure 3: Schematic view of the measurement system

Irradiating $\beta$ rays to the sample through a 4 $\mathrm{mm} \phi$ collimator, and the photomultiplier tube (PMT) detect photons.

\begin{tabular}{|l|}
\hline samples \\
\hline NIT $($ Film $) \sim 150 \mu \mathrm{m}$ thickness \\
gelatine film $(\mathrm{Gel}.) \sim 80 \mu \mathrm{m}$ thickness \\
\hline \hline radiation source \\
\hline${ }^{90} \mathrm{Sr} \sim 600 \mathrm{kBq}$ \\
\hline
\end{tabular}

Table 1: Information on used samples and radiation source

We used ${ }^{90} \mathrm{Sr}$ as radiation source and NIT as a main sample, and gelatine film as a reference since approximately $60 \%$ of Film consist of gelatin.

Irradiating $\beta$ rays to the sample through a $4 \mathrm{~mm} \phi$ collimator, and the photomultiplier tube (PMT) detect photons from the photon emission process mentioned in $\S 1$ if it was the case. The output signals from the PMT pass through the amplifier and integration circuit, and feed into mul-

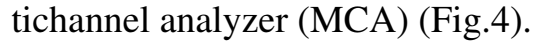

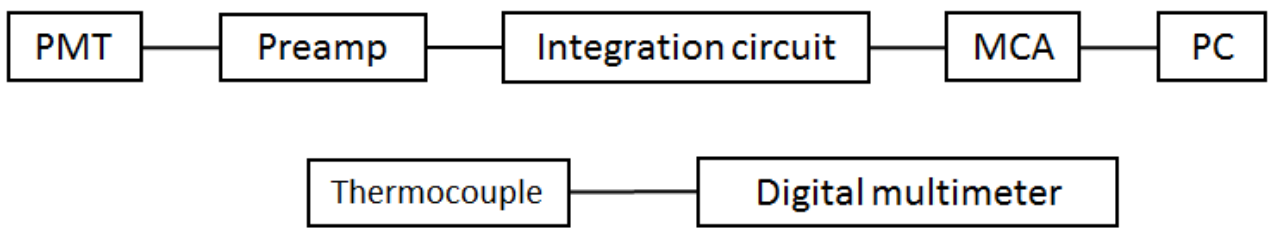

Figure 4: Block diagrams of the signal processing and temperature measurement system

The output signals from the PMT pass through the amplifier and integration circuit, and feed into multichannel analyzer (MCA).

The photon counting was performed for 20 seconds in every 2 minutes by MCA, started when the liquid nitrogen surface was $1 \mathrm{~cm}$ above from the sample surface. The MCA threshold was set to reduce thermal noise. 


\section{The results}

The measurement results are shown in the Fig.51. The horizontal and the vertical axis of the plots show elapsed time since liquid nitrogen poured and the number of signals counted for 20 seconds converted into the rate/second, respectively. Then, the error bars on the "time" are time duration of the measurement, i.e. 20s, and the error bars on the "rate" are the statistical error. At first, there are excess when placing RI, with and without Gel. (data points (c) and (d) ) compared with placing the sample only (data points (a) and (b) ) cases. This comes from effect of RI, because there is no any effect of placing Gel. Finally, obvious excess was seen when placing the RI and the Film (data points (e) ). The increasing tendency in the early stage (time $<500 \mathrm{~s}$ ) is thought that the low energy $\beta$ rays also can reach to the Film as the liquid nitrogen evaporating. At this measurement, the Film temperature has been maintained at liquid nitrogen temperature as shown in Fig6.

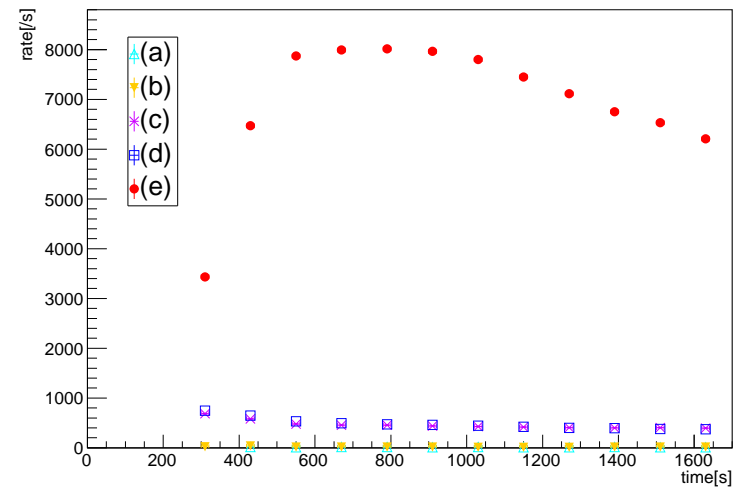

Figure 5: The results (a) - (e) show when placing Gel. only, Film only, RI only, Gel. and RI, and Film and RI, respectively.

Obvious excess points exist when placing the RI and the Film (data points (e) ).

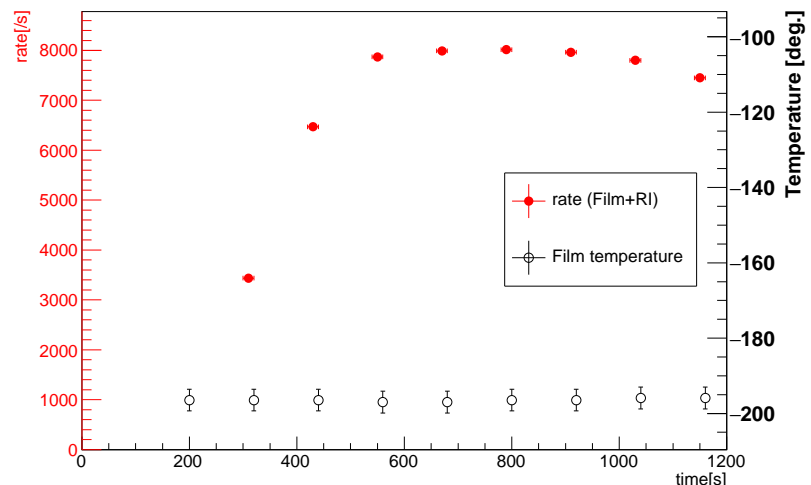

Figure 6: Plot of Film temperature at the measurement

Film temperature is maintained at liquid nitrogen temperature.

\section{Prospect}

We succeeded to detect photon emission from nuclear emulsion excited by $\beta$ rays at cryogenic temperature. There are several usages of this luminescence of the nuclear emulsions. First, it is useful for tagging the position and time of the event occurred in an experiment using nuclear emulsions. This enables us to analyze only limited volume of emulsion and it helps to realize large target mass in the experiments with low reaction rate, for example, the dark matter search experiment [3] . Of course, it may be possible to use nuclear emulsion as a scintillator by itself. In the future, we plan to characterize the emission caused by $\beta$ rays about the viewpoint of the number of photons and the light emission time constant, and would like to discriminate particles, $\alpha$ rays or $\beta$ rays. According to the result of Kimura et al. [四], the charged particle with high nuclear stopping power may cause local temperature rise when penetrating the $\mathrm{AgBr} \cdot \mathrm{I}$ crystals, and this temperature 
rise may also affect to the photon emission. From this point, it is expected that, $\alpha$ rays and nuclear recoil events can also be distinguished using the difference of the number of photons and the time constant decrease. of the luminescence Because particles with higher nuclear stopping power cause more dramatic temperature rise.Finally the information of emission photons can be a key input for understanding the properties of the $\mathrm{AgBr} \cdot \mathrm{I}$ crystals itself.

\section{References}

[1] S. Takada, Photogr.Sci.Eng.21,3 (1997)

[2] T. Nakamura, et al., Nucl. Instr. and Meth. A, 556, pp. 80 (2006)

[3] T. Naka, et al., EAS Publications Series, 53, pp. 51-58 (2012)

[4] M. Kimura, et al., Nuclear Instruments \& Methods in Physics Research A (2016) 